\title{
MYOD1-mutant spindle cell and sclerosing rhabdomyosarcoma: an aggressive subtype irrespective of age. A reappraisal for molecular classification and risk stratification
}

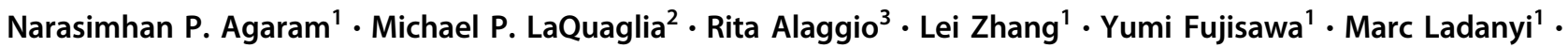 \\ Leonard H. Wexler ${ }^{4}$. Cristina R. Antonescu'
}

Received: 17 May 2018 / Revised: 24 July 2018 / Accepted: 28 July 2018 / Published online: 4 September 2018

(c) United States \& Canadian Academy of Pathology 2018

\begin{abstract}
Sclerosing and spindle cell rhabdomyosarcoma is a rare histologic subtype, designated in the latest WHO classification as a stand-alone pathologic entity. Three genomic groups have been defined: an infantile subset of spindle cell rhabdomyosarcoma harboring VGLL2-related gene fusions, a MYOD1-mutant subset commonly associated with sclerosing morphology, and a subset lacking recurrent genetic abnormalities. In this study, we focus on MYODl-mutant rhabdomyosarcoma to further define their clinicopathologic characteristics and behavior in a larger patient cohort. We investigated 30 cases of MYODI-mutant rhabdomyosarcoma (12 previously reported and 18 newly diagnosed) with an age range of 2-94 years, including 15 children. All cases showed morphology within the spectrum of spindle cell/sclerosing rhabdomyosarcoma ( 8 cases showing pure sclerosing morphology, 8 cases showing pure spindle cell morphology and 14 cases showing a hybrid phenotype of spindle, sclerosing and primitive undifferentiated areas). All tumors harbored either homozygous or heterozygous MYODI (p.L122R) exon 1 mutations. In 10 (33\%) cases, a co-existent PIK3CA mutation was identified. Hot-spot mutations in NRAS and HRAS were each identified in a single case, respectively. Follow-up was available on $22(73 \%)$ patients with a median duration of 28 months. Local recurrence was seen in $12(55 \%)$ and distant recurrence in $12(55 \%)$ cases, despite multimodality chemoradiation therapy. At last follow-up, $15(68 \%)$ patients died of the disease, one patient was alive with disease and five had no evidence of disease. The prognosis was equally poor in pediatric and adult patients. In conclusion, MYOD1 mutation defines an aggressive rhabdomyosarcoma subset, with poor outcome and response to therapy, irrespective of age. Given that this distinct molecular subtype is characterized by an aggressive biologic behavior compared to other genetic subtypes of spindle and sclerosing rhabdomyosarcoma, the MYODl genotype should be used as a molecular marker in both subclassification and prognostication of rhabdomyosarcoma.
\end{abstract}

Electronic supplementary material The online version of this article (https://doi.org/10.1038/s41379-018-0120-9) contains supplementary material, which is available to authorized users.

Narasimhan P. Agaram

agaramn@mskcc.org

$\triangle$ Cristina R. Antonescu antonesc@mskcc.org

1 Department of Pathology, Memorial Sloan Kettering Cancer Center, New York, NY, USA

2 Department of Pediatric Surgery, Memorial Sloan Kettering Cancer Center, New York, NY, USA

3 Department of Pathology, Children's Hospital of Pittsburgh of UPMC, Pittsburgh, PA, USA

4 Department of Pediatrics, Memorial Sloan Kettering Cancer Center, New York, NY, USA

\section{Introduction}

Rhabdomyosarcoma is the most common soft tissue sarcoma in children, accounting for $40 \%$ of all pediatric soft tissue sarcoma [1], while rhabdomyosarcoma in adults is far less common. The latest WHO classification of soft tissue tumors [2] classifies rhabdomyosarcoma into four subtypes-embryonal rhabdomyosarcoma, alveolar rhabdomyosarcoma, spindle cell/sclerosing rhabdomyosarcoma, and pleomorphic rhabdomyosarcoma. While alveolar rhabdomyosarcoma follows a highly aggressive course, the prognosis of embryonal rhabdomyosarcoma has significantly improved in recent years with an overall survival of $70 \%$ at 5 years for patients presenting with localized disease [3]. Spindle cell/sclerosing subtype was only recently separated from the embryonal 
rhabdomyosarcoma group and defined as a stand-alone pathologic entity based on its distinctive morphologic features. Spindle cell variant of rhabdomyosarcoma was initially described in children in the paratesticular and head and neck locations, being associated with a more favorable prognosis $[4,5]$. A subset of spindle cell rhabdomyosarcoma was shown to have prominent hyaline sclerosis and pseudo-vascular growth pattern, suggesting morphologic overlap with the even less common sclerosing type rhabdomyosarcoma [6]. As both spindle cell and sclerosing rhabdomyosarcoma have similar clinical presentations, Mentzel and colleagues suggested a histologic spectrum of a single pathologic entity [7-9]. More recently, a recurrent MYOD1 L122R mutation was identified as a frequent genetic event in adult spindle cell rhabdomyosarcoma, by two independent groups $[10,11]$ and found to be associated with altered function of MYOD1 and having an aggressive clinical course [11]. Subsequent studies from our group and others have identified similar hot-spot MYOD1 mutations in pediatric rhabdomyosarcoma with spindle cell/sclerosing morphology and confirmed the association with poor clinical outcomes [12-16]. Here, we describe the largest cohort of MYOD1 mutant rhabdomyosarcoma to date, spanning various age groups and clinical presentations. As rhabdomyosarcoma with MYOD1 mutation is associated with a highly lethal outcome despite multimodality chemoradiation therapy, it raises an argument for a molecular subclassification of the spindle cell/sclerosing rhabdomyosarcoma based on their specific genetic signatures, and to include this variant as a separate subtype, with an unfavorable behavior comparable to alveolar rhabdomyosarcoma.

\section{Materials and methods}

\section{Patient selection and histologic diagnosis}

Archival and personal consultation material (CRA) from adult and pediatric patients with diagnosis of spindle and sclerosing rhabdomyosarcoma was retrieved from the pathology files at Memorial Sloan Kettering Cancer Center, in which additional material was available for MYOD1 mutation testing. Thirty cases were identified in which the diagnosis of spindle and sclerosing rhabdomyosarcoma was confirmed by re-review of histologic slides and the presence of a MYOD1 mutation was identified. Formalin fixed paraffin embedded tissue was available on all the cases selected for the study. Twelve cases were previously included in the following studies by our group: 9 cases in the study by Agaram et al. [13] which included 2 cases reported in Kohsaka et al. [11], 10 cases in the study by Alaggio et al. [14], and 3 cases in Owosho et al. [15]. This information is also detailed in Table 1 . The remaining 18 cases are being genetically examined for the first time. The study was approved by the Institutional Review Board at Memorial Sloan Kettering Cancer Center. The morphologic features were characteristic of those described in spindle cell/sclerosing rhabdomyosarcoma [4-6, 8, 17], and showed a relatively monomorphic spindle cell proliferation arranged in long, intersecting fascicles, in a background of variably fibrotic stromal component. The tumors typically had an infiltrative growth within the adjacent skeletal muscle and adipose tissue. The nuclear pleomorphism was mild, while lacking evidence of rhabdomyoblastic differentiation. The immunohistochemical profile was based on desmin reactivity, typically diffuse and strong, at least focal reactivity for myogenin and diffuse positivity for MYOD1.

\section{PCR and sanger sequencing}

Genomic DNA was isolated either from fresh-frozen or from archival paraffin tissue, as described previously [18] in 19 samples. Targeted PCR was performed for the known MYODI exon 1 hot-spot mutation and PIK3CA exons 9 and 20 mutations, using the following primer sequences: MYOD1 Ex1 fwd: 5'-CCTACTGTGGGCCTGCAAG-3' and Ex1 rev: 5'-GGATCTCCACCTTGGGCAAC-3', PIK3CA-Ex9 fwd: 5'-CCAGAGGGGAAAAATATGA CAAAG-3', PIK3CA-Ex9 rev: 5'-CCATTTTAGCACT TACCTGTGACTCC-3', PIK3CA-Ex20 fwd: 5'CTCAATGATGCTTGGCTCTGG-3' ${ }^{\prime}$ and PIK3CA-Ex20 rev: 5'-GTGGAATCCAGAGTGAGCTTTC-3', using Clontech advantage 2 PCR KIT, at $64.5^{\circ} \mathrm{C}$ annealing temperature for 35 cycles. Direct sequencing of PCR products was performed and compared to the NCBI human MYODI and PIK3CA gene sequences.

\section{MSK-IMPACT assay}

Details of the MSK-IMPACT assay have been previously published [19]. Briefly, MSK-IMPACT is a comprehensive molecular profiling assay that involves hybridization capture and deep sequencing of all exons and selected introns of up to 468 oncogenes and tumor-suppressor genes, allowing the detection of point mutations, small and large insertions or deletions, and rearrangements. In addition to capturing all coding regions of the genes, the assay also captures $>1000$ intergenic and intronic singlenucleotide polymorphisms (tiling probes), interspersed homogenously across the genome, aiding the accurate assessment of genome-wide copy number. In total, the probes target $\sim 1.5$ megabases of the human genome. 
Table 1 Clinicopathologic and molecular features and followup of MYOD1-mutant rhabdomyosarcoma

\begin{tabular}{|c|c|c|c|c|c|c|c|c|c|c|}
\hline Case \# & Age & Location & Morphology & $\begin{array}{l}\text { MYOD1 Ex1 } \\
\text { p.L122R }\end{array}$ & PIK3CA & Therapy & LR & DR & $\begin{array}{l}\text { FU duration } \\
\text { (months) }\end{array}$ & FU status \\
\hline $1^{\mathrm{a}}$ & $2 / \mathrm{F}$ & Buttock & Sp, Pri & Heterozygous & WT & Chemo+RT & & DR & 12 & DOD \\
\hline 2 & $4 / \mathrm{F}$ & Calf & Scl, Pri & Heterozygous & WT & & & & na & na \\
\hline $3^{b}$ & $7 / \mathrm{F}$ & I-abd & Scl & Homozygous & WT & Chemo+RT & LR & DR & 28 & DOD \\
\hline $4^{\mathrm{ab}}$ & 8/M & Thigh & $\begin{array}{l}\text { Scl, Pri (pre-Rx); } \\
\text { Sp (post-Rx) }\end{array}$ & Homozygous & WT & Chemo & & & 44 & NED \\
\hline $5^{\mathrm{a}}$ & $9 / \mathrm{F}$ & $\mathrm{H} \& \mathrm{~N}$ & $\mathrm{Sp}$ & Homozygous & WT & & LR & & 12 & DOD \\
\hline $6^{\mathrm{a}}$ & 9/M & $\mathrm{H} \& \mathrm{~N}$ & $\mathrm{Sp}$ & Homozygous & WT & & LR & & 42 & DOD \\
\hline 7 & $9 / \mathrm{F}$ & $\mathrm{H} \& \mathrm{~N}$ & $\mathrm{Scl}$ & Homozygous & E545A & & & & na & na \\
\hline $8^{a}$ & $10 / \mathrm{F}$ & Paraspinal & $\mathrm{Sp}$ & Homozygous & WT & Chemo+RT & LR & DR & 35 & DOD \\
\hline $9^{\mathrm{ac}}$ & $10 / \mathrm{F}$ & Buttock & Scl, Pri & Homozygous & G1049R & & & DR & 6 & DOD \\
\hline 10 & $10 / \mathrm{F}$ & $\mathrm{H} \& \mathrm{~N}$ & $\mathrm{Scl}$ & Heterozygous & WT & Chemo+RT & LR & & 48 & NED \\
\hline $11^{\mathrm{a}}$ & $11 / \mathrm{F}$ & $\mathrm{H} \& \mathrm{~N}$ & $\mathrm{Scl}$ & Homozygous & Q546R & & & & na & na \\
\hline $12^{\mathrm{a}}$ & $13 / \mathrm{F}$ & Chest wall & $\begin{array}{l}\text { Scl (pre-Rx); Sp } \\
(\text { post-Rx) }\end{array}$ & Homozygous & E545K & Chemo+RT & LR & DR & 21 & DOD \\
\hline $13^{\mathrm{a}}$ & $15 / \mathrm{F}$ & $\mathrm{H} \& \mathrm{~N}$ & $\mathrm{Scl}$ & Homozygous & E542K & Chemo & LR & DR & 26 & DOD \\
\hline $14^{\mathrm{a}}$ & $17 / \mathrm{M}$ & Paravertebral & $\mathrm{Sp}, \mathrm{Scl}$ & Homozygous & WT & & & DR & 24 & DOD \\
\hline $15^{b}$ & $17 / F$ & Thorax & $\mathrm{Sp}$ & Homozygous & WT & Chemo+RT & LR & DR & 68 & DOD \\
\hline $16^{\mathrm{b}}$ & $21 / \mathrm{M}$ & Pelvis & $\mathrm{Sp}$ & Homozygous & WT & Chemo & LR & DR & 42 & DOD \\
\hline $17^{\mathrm{b}}$ & $21 / \mathrm{F}$ & $\mathrm{H} \& \mathrm{~N}$ & $\mathrm{Sp}, \mathrm{Scl}$, Pri & Homozygous & M1043V & Chemo+RT & & & 30 & NED \\
\hline 18 & $21 / \mathrm{F}$ & $\mathrm{H} \& \mathrm{~N}$ & $\mathrm{Sp}, \mathrm{Scl}$ & Heterozygous & - & & & & na & na \\
\hline $19^{\mathrm{b}}$ & 26/M & Lower leg & $\mathrm{Sp}, \mathrm{Scl}$ & Homozygous & H1047R & Chemo & & & 4 & NED \\
\hline $20^{\mathrm{b}}$ & $31 / \mathrm{F}$ & $\mathrm{H} \& \mathrm{~N}$ & $\mathrm{Scl}$ & Homozygous & $\mathrm{K} 111 \mathrm{E}$ & Chemo+RT & LR & & 12 & AWD \\
\hline $21^{\mathrm{a}}$ & $32 / \mathrm{M}$ & $\mathrm{H} \& \mathrm{~N}$ & $\mathrm{Sp}, \mathrm{Scl}$ & Heterozygous & WT & Chemo+RT & LR & & 65 & DOD \\
\hline 22 & $33 / \mathrm{M}$ & Thigh & $\mathrm{Sp}$ & Homozygous & WT & & & & na & na \\
\hline $23^{\mathrm{a}}$ & $34 / \mathrm{F}$ & $\mathrm{H} \& \mathrm{~N}$ & $\mathrm{Sp}, \mathrm{Scl}$ & Heterozygous & E542V & Chemo & & DR & 42 & DOD \\
\hline $24^{\mathrm{b}}$ & $36 / \mathrm{M}$ & Forearm & $\mathrm{Sp}$ & Homozygous & K111 del & Chemo+RT & LR & DR & 16 & DOD \\
\hline 25 & $38 / \mathrm{F}$ & Leg & $\mathrm{Sp}$ & Homozygous & WT & & & & na & na \\
\hline 26 & 39/M & Lower leg & $\mathrm{Sp}, \mathrm{Scl}$ & Heterozygous & WT & Chemo & & & 60 & NED \\
\hline $27^{\mathrm{b}}$ & $44 / \mathrm{F}$ & Paraspinal & $\mathrm{Sp}$ & Homozygous & WT & Chemo+RT & & & 13 & NED \\
\hline $28^{b}$ & $45 / \mathrm{M}$ & Liver & Pri, Sp & Heterozygous & WT & & & & na & na \\
\hline 29 & $77 / \mathrm{M}$ & Lower leg & $\mathrm{Sp}, \mathrm{Scl}$ & Homozygous & WT & Chemo & & DR & 32 & DOD \\
\hline 30 & 94/M & Lower leg & $\mathrm{Scl}$ & Homozygous & WT & & & & na & na \\
\hline
\end{tabular}

$M$ male, $F$ female, $F U$ follow-up, $H \& N$ head and neck, intra-abd intra-abdominal, $S p$ spindle, $S c l$ sclerosing, Pri primitive, $L R$ local recurrence, $D R$ distant recurrence, $N E D$ no evidence of disease, $A W D$ alive with disease, $D O D$ dead of disease, $R T$ external beam radiation, $n a$ not available

${ }^{\mathrm{a} C}$ Cases previously included in references [11-15]

${ }^{\mathrm{b}}$ Cases tested by MSK-IMPACT

${ }^{\mathrm{c}}$ Cases tested by RNAseq

\section{Results}

\section{Clinicopathologic features}

The clinicopathologic features are summarized in Table 1. There were 30 patients, including 18 females and 12 males, ranging in age from 2 to 94 years (median age-19 years). The cohort included $15(50 \%)$ children (12 females, 3 males), ranging in age from 2 to 17 years, and $15(50 \%)$ adults (9 males, 6 females) with an age range of 21-94 years. The anatomic location of the tumors varied, including: head and neck (11), trunk/abdominal (paraspinal-3, chest wall-1, thorax-1, pelvic/intra-abdominal-2), extremities (lower leg-6, buttock-2, thigh-2, forearm1 ), and visceral (liver-1).

Histologically, all cases showed typical spindle cell/ sclerosing morphology as previously described [13]. Eight cases showed pure sclerosing rhabdomyosarcoma 

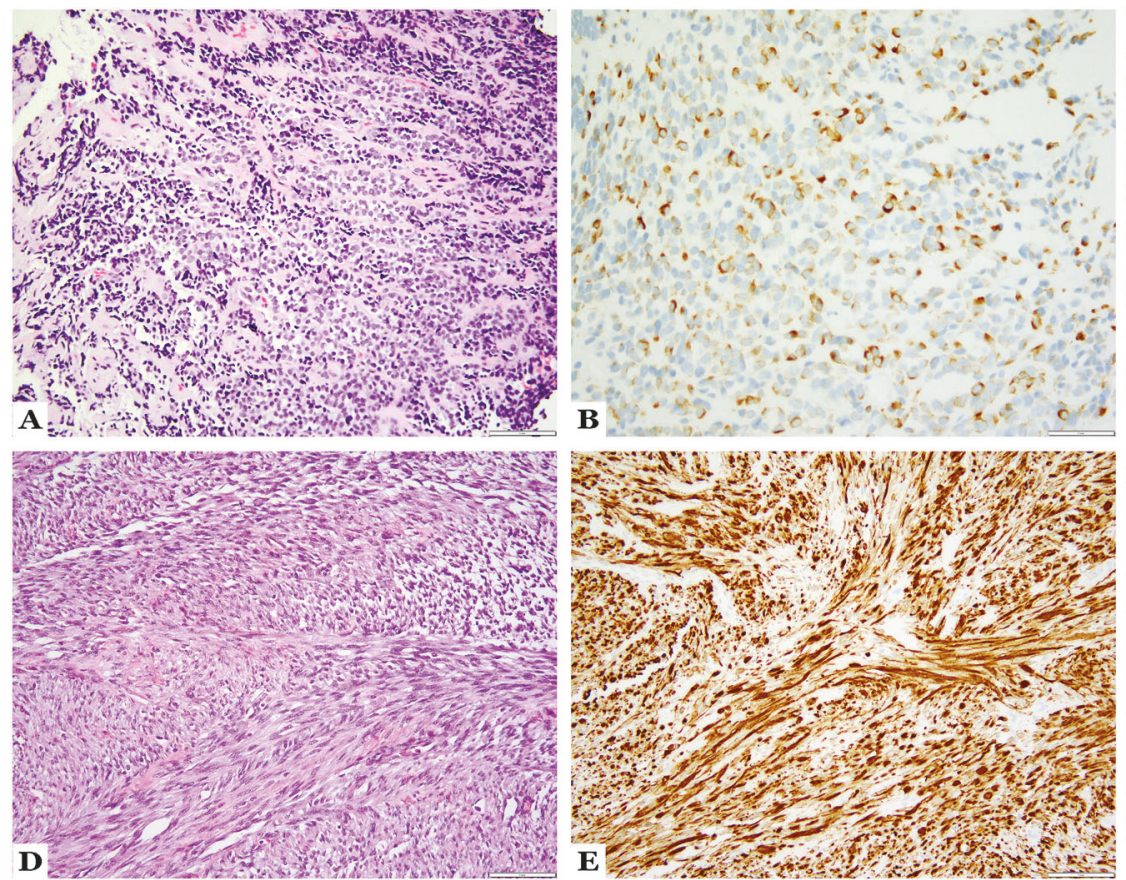

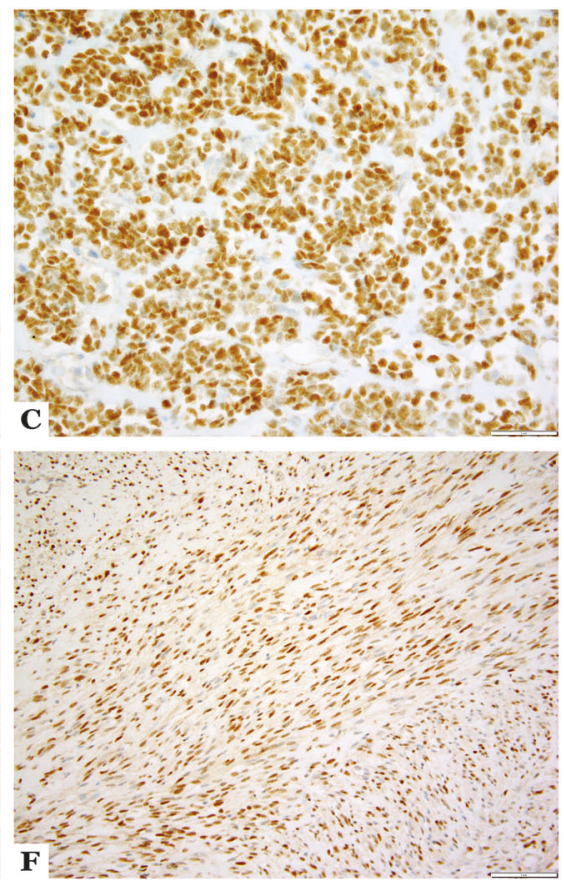

(c). d-f Case 21 showing spindle cell morphology with intersecting fascicles of monomorphic fusiform cells (d), immunohistochemical stains for desmin with strong cytoplasmic positivity (e), and MYOD1 showing diffuse nuclear positivity (f)

(Fig. 2) were seen in five cases. In two of these cases (cases 1 and 28), there was associated spindle cell component, two cases (cases 2 and 4) showed associated sclerosing component and one case (case 17) had both sclerosing and spindle cell components.

Mitotic activity was high in most tumors ranging from 10 to 25 per 10 high-power fields. Numerous apoptotic bodies were identified in most cases. Necrosis was an uncommon feature being present in four cases (cases 1, 9, 14, and 28), as focal and spotty in three and as geographic areas of necrosis in one (case 1, Fig. 2).

Immunohistochemical staining profile was available for review in 27 of the 30 cases. Desmin was diffusely positive in all cases, while myogenin was focally positive in all but three of the tumors (cases 4, 7, and 17). MYOD1 immunostaining was performed in 16 cases and was diffusely positive in all of the tumors tested (Fig. 1).

A subset of cases showed morphologic changes in the post-therapy resection when compared to the pre-therapy biopsy (Fig. 3). Cases 4 and 12 showed primitive and sclerosing morphology on the pre-treatment biopsy, and on post-therapy resections, showed predominantly spindle cell morphology with areas of maturation with rhabdomyoblastic differentiation. Cases 23 and 26 

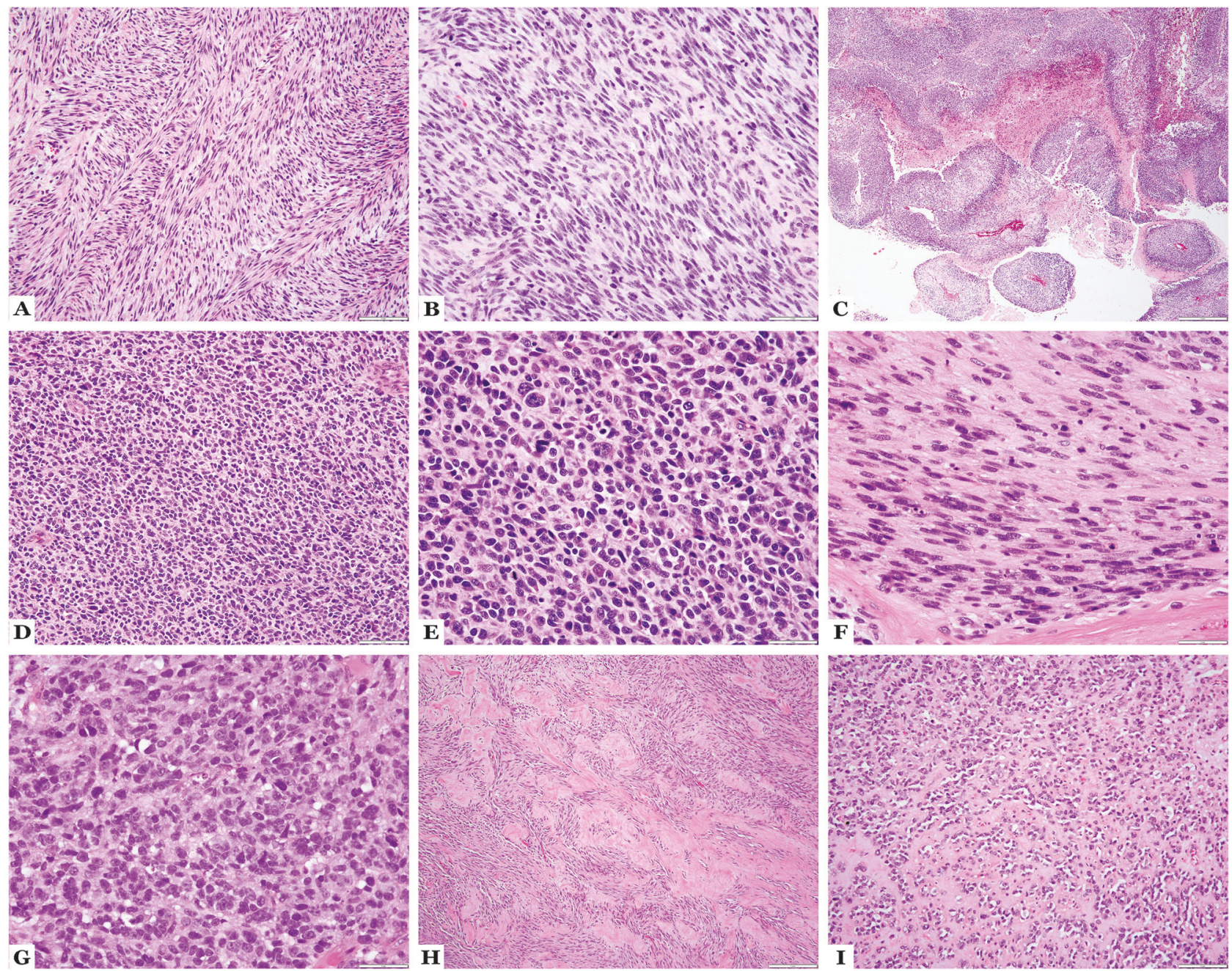

Fig. 2 MYOD1-mutant rhabdomyosarcoma with primitive round to spindle cell component. a-c Case 1 showing areas of classic spindle cell morphology with fascicular growth (a), solid zones of primitive appearing spindle cell component (b) and geographic necrosis (MPNST-like); d-f Case 28 showing tumor in the liver with

showed spindle, sclerosing, and rhabdomyoblastic differentiation in the post-treatment samples.

\section{p.L122R MYOD1 mutations present in both sclerosing and spindle cell rhabdomyosarcoma}

Nineteen cases were analyzed by PCR for mutations in MYOD1 exon 1. All cases showed a p.L122R (c. T365G) mutation. Targeted exome sequencing by MSK-IMPACT showed MYODI L122R mutations in 10 cases. In one case (case 9), previously reported by Alaggio et al. [14], RNA sequencing data analyzed by mutation detection algorithms showed MYOD1 L122R mutations. Overall, 22 cases (73\%) showed homozygosity for MYOD1 L122R presumably via loss of the other allele, while the remaining $8(27 \%)$ were heterozygous for the MYOD1 L122R mutation. undifferentiated primitive round cell component $(\mathbf{d}, \mathbf{e})$ and areas of spindling (f); $\mathbf{g}-\mathbf{i}$ Case 23, a post-therapy resection, showing primitive round cell areas (g), spindle cell morphology (h) and areas with sclerosing morphology (i). (H\&E stains)

\section{Concurrent PIK3CA, NRAS, HRAS mutations are present in MYOD1-mutant rhabdomyosarcoma}

A subset of MYOD1-mutant rhabdomyosarcoma harbor co-existing PIK3CA mutations, either in the helical domain (E542K, E545K) or in the kinase domain (H1047R) [11, 13]. We investigated mutations at these PIK3CA hot spots in all except one case. Ten (33\%) cases showed coexistent PIK3CA mutations, two in exons 2 (K111del., K111E), five in exon 9 (E542K, E542V, E545K, E545A, Q546R), and three cases in exon 20 (H1047R, M1043V), respectively (Table 1). Interestingly, 6 of the 10 cases with co-existent PIK3CA mutations showed pure sclerosing morphology, 3 cases showed hybrid spindle cell/sclerosing morphology, and only 1 case showing pure spindle cell morphology. 


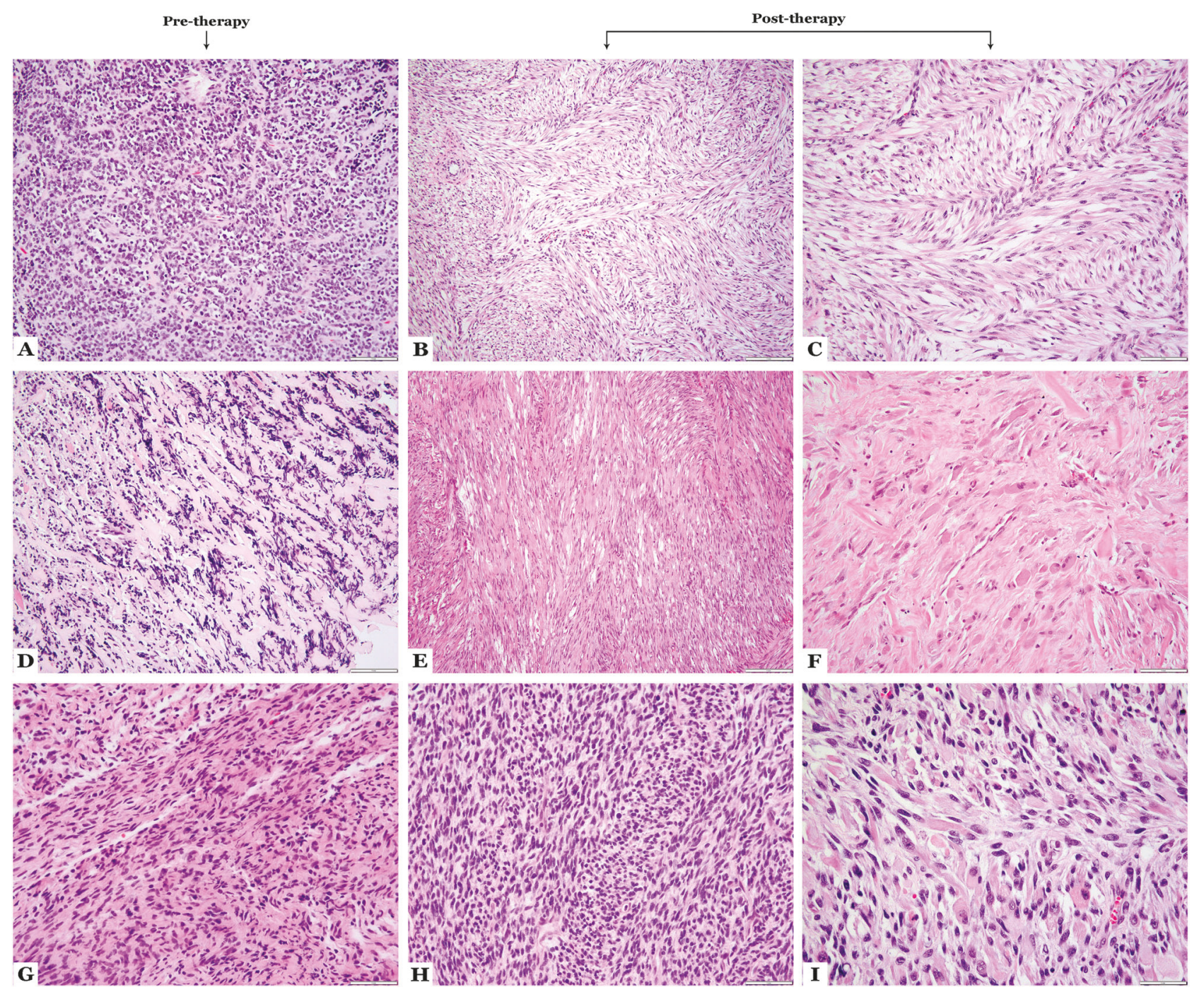

Fig. 3 Morphologic spectrum of MYOD1-mutant rhabdomyosarcoma following therapy. a-c Case 4 showing sclerosing areas on the pretherapy biopsy (a), while in the post-therapy resection $(\mathbf{b}, \mathbf{c})$ showing pure spindle cell morphology; d-f Case 12 showing a sclerosing morphology in the pre-therapy biopsy (d), while the post-therapy

Additionally, mutually exclusive to PIK3CA mutated cases, one case each showed coexistent NRAS (Q61L) (case 3) and HRAS (G13R) (case 21) hot-spot mutations, respectively.

\section{Targeted exome sequencing identifies additional genetic abnormalities in MYOD1-mutant rhabdomyosarcoma}

Apart from the MYODI mutations in exon 1, targeted exome sequencing (MSK-IMPACT) showed additional genetic alterations in the 10 cases analyzed, mainly gene copy number alterations and mutations (Fig. 4, Supplementary Table 1). Case 16 showed low-level amplification of the MYODl gene in addition to the exon $1 \mathrm{~L} 122 \mathrm{R}$ resection reveals a spindle cell morphology (e) and areas of maturation with rhabdomyoblastic morphology (f); $\mathbf{g}$-i Case 24 showing spindle cell morphology on pre-therapy biopsy (g), while post-therapy resection shows cellular spindle cell areas (h) and areas of maturation (i). (H\&E stains)

MYODI gene mutation. Interestingly, we identified another spindle cell rhabdomyosarcoma which showed amplification of the MYODl gene, although it lacked MYOD1 mutations. This case (not included in the study group) occurred in a 74-year-old patient with a hard palate lesion who recurred and succumbed of disease despite chemotherapy and radiation 12 months since diagnosis.

IGF2 copy number gain/amplification was a frequent genetic alteration, being identified in $4(40 \%)$ cases (cases $15,16,20$, and 27). FISH studies confirmed amplification in cases 16 and 27 (results not shown), while no IGF2 amplification could be confirmed in the remaining two cases, suggesting low-level gains or spatial heterogeneity.

$M D M 2$ gene amplification was seen in two cases (cases 24 and 28). Case 9 also showed co-existent FGFR4 
Fig. 4 Oncoprint image showing the variant genetic alterations identified in the study cases

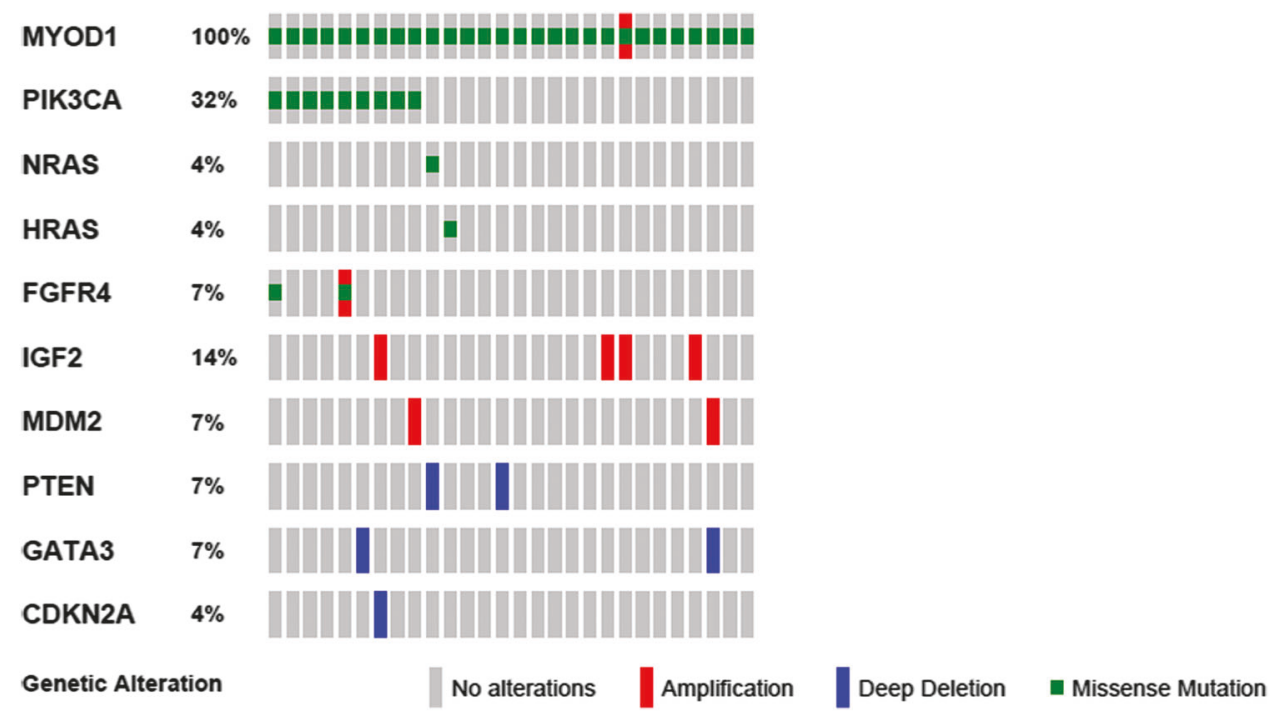

(V548M) mutation. Case 17 showed the presence of both amplification and a V550L mutation in the FGFR4 gene. Losses (deletions) of tumor suppressor genes, such as PTEN (cases 3 and 4), GATA3 (cases 19 and 28), and CDKN2A/ $C D K N 2 B$ (case 20) were also noted. No TP53 gene alterations were identified.

\section{MYOD1-mutated rhabdomyosarcoma follows a highly aggressive clinical course in both children and adults}

Follow-up information (Table 1) was available in 22 of the 30 cases $(73 \%)$, with duration of 4-68 months (mean28.8 months, median-28 months). Eleven of the patients were treated with a combination of chemotherapy and radiotherapy, and seven patients were treated with chemotherapy alone. Twelve (55\%) patients developed local recurrence and 12 (55\%) developed distant recurrence, including 7 (32\%) patients who developed local recurrence and distant recurrence. The sites of distant recurrences included lung, mediastinum, breast, abdominal soft tissue, spleen, and chest wall. At last follow-up, 6 (27\%) patients had no evidence of disease, $1(5 \%)$ patient was alive with disease, and $15(68 \%)$ patients died of the disease at 12-68 months following diagnosis. Follow-up was not available on seven cases and one was a recent case. Based on the available follow-up, the 3-year and 4-year overall survival was $36 \%$ and $18 \%$, respectively.

Ten of the $12(83 \%)$ pediatric cases with follow-up data available died of the disease, and two had no evidence of disease. Of the nine adult cases with follow-up data available, $5(55 \%)$ died of the disease, one is alive with disease and three had no evidence of disease. Survival analysis showed no statistical difference of survival outcomes for pediatric and adult subgroups (Supplementary Figure 1) and for cases with and without PIK3CA mutations (Supplementary Figure 2).

\section{Discussion}

MYOD1 mutations in rhabdomyosarcoma were initially reported in 2014 by two independent groups [10, 11]. In order to investigate the possibility of a shared pathogenesis among the spindle cell and sclerosing rhabdomyosarcoma, spanning both age groups, our group investigated an initial cohort of 16 pediatric and adult spindle cell and sclerosing rhabdomyosarcoma [13], of which 56\% (9/16 cases) showed MYOD1 mutations. All 5 rhabdomyosarcomas with sclerosing histology, presenting either in pediatric or in adult age-group, were positive for MYOD1 gene mutations, while only 4 (36\%) of 11 spindle cell rhabdomyosarcomas showed $M Y O D 1$ mutations, 2 each in children and adults. In contrast to the study by Szuhai et al. [10] where only homozygous MYOD1 mutations were identified, 4/9 mutant tumors in our study showed MYOD1 p.L122R heterozygous mutations, while the remaining were homozygous. Thus our initial findings confirmed that spindle cell and sclerosing rhabdomyosarcomas share genetic abnormalities thereby providing a strong molecular basis in supporting the classification of these two groups as a single pathologic entity. Furthermore, MYODI mutations were consistently identified in rhabdomyosarcoma displaying sclerosing morphology, regardless of age at presentation, in keeping with a homogeneous genetic entity.

Subsequently, focusing only on the pediatric-age group, our group identified three distinct molecular subsets of the spindle cell/sclerosing rhabdomyosarcoma [14]. The first, so-called 'congenital or infantile spindle cell 
rhabdomyosarcoma', encompassing tumors presenting at birth or within one year of age, with predilection for the trunk, and harboring recurrent gene fusions, involving critical transcriptional activators of muscle-specific genes, such as VGLL2, TEAD1, and SRF [14, 20]. These patients followed a favorable clinical outcome, lacking metastatic potential, all being alive and well at long-term follow-up. These fusion-positive infantile spindle cell rhabdomyosarcoma group appeared to closely resemble the behavior of ETV6-NTRK3-positive infantile fibrosarcomas, and we argued that the findings militated against their classification as a 'high grade neoplasm' and suggested downgrading of their current therapy and possibly excluding the need for chemotherapy if completely excised. The second group was that of 'MYOD1-mutant spindle cell/sclerosing rhabdomyosarcoma', with or without accompanying PIK3CA mutations, occurring in older children, and following a highly aggressive course with high mortality despite multimodality therapy. In that study, MYODI mutations were the most common genetic abnormality in pediatric spindle cell/sclerosing rhabdomyosarcoma, occurring in $64 \%$ of children beyond one year of age, and suggesting that the MYOD1 mutation can be used as a molecular biomarker to stratify these high risk patients. The third group was the remaining 'genetically negative' group, lacking gene fusions or $M Y O D 1$ mutations, often presenting intraabdominally or in the genito-urinary area. This molecularnegative group followed a favorable clinical course and might represent embryonal rhabdomyosarcoma with spindle cell areas.

As head and neck is the most common anatomic site for spindle and sclerosing rhabdomyosarcoma, our group reported 13 cases of spindle cell/sclerosing rhabdomyosarcoma arising in this location, including three tumors with MYOD1 mutations, all showing sclerosing histology [15]. When comparing the outcome of all histologic subtypes in a cohort of 99 rhabdomyosarcomas from the head and neck region, alveolar rhabdomyosarcoma and MYOD1-mutant spindle cell/sclerosing rhabdomyosarcomas had similar poor prognosis [12]. In fact, the 5-year overall survival rate for embryonal rhabdomyosarcoma patients was significantly higher $(82 \%)$ compared to alveolar rhabdomyosarcoma $(53 \%)$ and spindle/sclerosing rhabdomyosarcoma (50\%). More specifically, the MYOD1-mutant positive sclerosing rhabdomyosarcoma had a 30\% 5-year overall survival compared to $75 \%$ of the spindle cell rhabdomyosarcoma [12].

The aim of the current study was to better define the demographics, pathologic features, and behavior in a large cohort of MYOD1 mutant positive rhabdomyosarcoma. With 30 cases (12 previously reported and 18 newly diagnosed cases), this is the largest patient cohort of MYOD1 mutant rhabdomyosarcoma to date. Our results establish
MYOD1 mutation as a surrogate biomarker of poor outcome regardless of age at presentation and phenotype (spindle, sclerosing, mixed). The study included 15 children and 15 adults, with a wide age range at diagnosis, the youngest being 2 years and the oldest being 94 years of age. In children, there was a female predilection, with 12 of the 15 cases occurring in girls. In adults, there was a fairly equal distribution for males and females. In keeping with prior literature, the head and neck location was the most common site, occurring in one-third $(33 \%)$ of patients, with equal predilection in both children and adults. The other common sites included the extremities and the trunk. At the molecular level, most $(22 / 30,73 \%)$ tumors harbored a MYOD1 homozygous mutation in exon 1 (p.L122R), while the remaining $37 \%$ of cases had a heterozygous genotype. This hot-spot mutation was equally detected by either Sanger sequencing or by the MSK-IMPACT targeted cancer gene panel, a next-generation molecular analysis using pairedend sequencing. An advantage of the latter approach is the identification of co-existing mutations, such as NRAS, HRAS, FGFR4, etc.

Shukla et al. [21] initially reported the presence of PIK3CA mutations in a small subset $(5 \%, 3 / 60)$ of rhabdomyosarcoma patients. Subsequently, Kohsaka et al. [11] noted an apparent association between the MYOD1 L122R mutation and concurrent mutations in PIK3CA hot spots (E542K, E545K, H1047R), suggesting that PIK3CA-mutated rhabdomyosarcoma should be screened for the MYODI mutation. In our initial study [13], three of the nine MYOD1-mutant spindle cell/sclerosing rhabdomyosarcoma showed helical domain PIK3CA mutations (E542K, E542V, and E545K), all showing a sclerosing phenotype (two

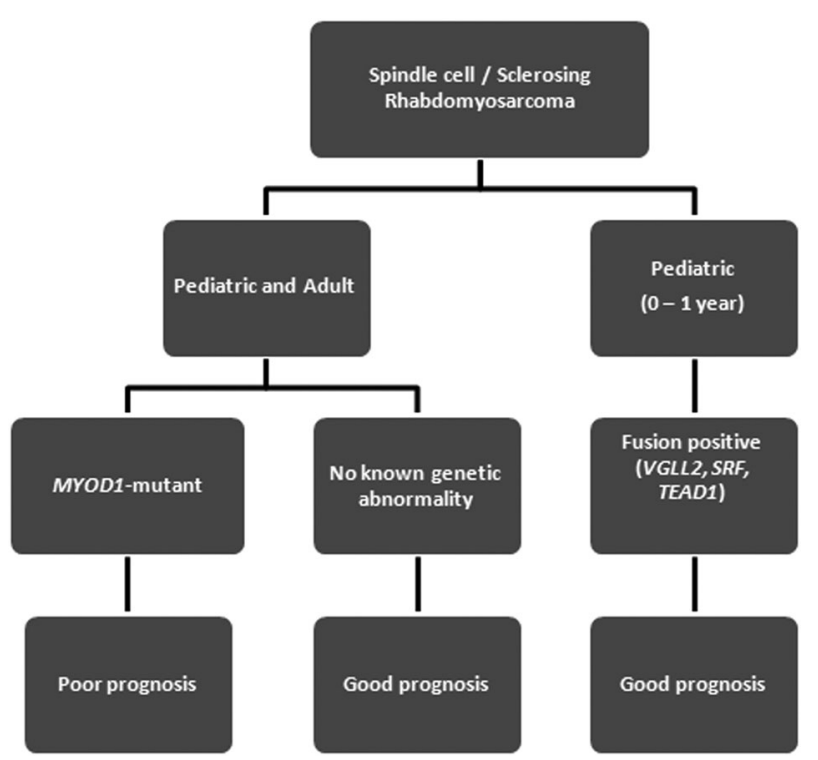

Fig. 5 Schematic diagram of spindle cell/sclerosing rhabdomyosarcoma genetic subclassification 
Table 2 Schematic showing the prognostic classification of rhabdomyosarcoma based on the current prognostic information $\mathrm{Sp} / \mathrm{Scl} \mathrm{RMS}$ spindle cell/ sclerosing rhabdomyosarcoma
Rhabdomyosarcoma

\begin{tabular}{lll}
\hline Poor prognosis & Good prognosis & Very good prognosis \\
\hline $\begin{array}{l}\text { 1. Alveolar rhabdomyosarcoma } \\
\text { 2. MYOD1-mutant Sp/Scl RMS }\end{array}$ & $\begin{array}{l}\text { 1. Embryonal rhabdomyosarcoma } \\
\text { 2. Sp/Scl RMS with no genetic } \\
\text { 3. Pleomorphic }\end{array}$ & $\begin{array}{l}\text { 1. Infantile Sp RMS with } \\
\text { rhabdomyosarcoma }\end{array}$ \\
\hline
\end{tabular}

pediatric and one adult). None of tumors lacking $M Y O D 1$ mutations showed PIK3CA mutations. Interestingly, in the study by Rekhi et al. [16], none of the 10 MYOD1 mutant cases had co-existent PIK3CA mutations. In the current series, $33 \%$ of cases showed co-existing PIK3CA mutations with a roughly even distribution between pediatric and adult cases. In one case, reported previously by Alaggio et al., whole transcriptome sequencing identified coexistent mutations in PIK3CA and FGFR4 genes. In addition to the previously reported hot-spot mutations in exons 9 and 20, one case showed a previously unreported PIK3CA exon 9 mutation (E545A) and two cases showed genetic abnormalities in exon 2 (K111E and K111del). Nine of the 10 (90\%) tumors with coexistent PIK3CA mutations showed sclerosing morphology, with 6 showing pure sclerosing pattern.

Another interesting finding is the identification of coexisting mutations in the RAS pathway, with one case each showing an NRAS (Q61L) and HRAS (G13R) hot-spot mutation, respectively. The clinical significance of these coexistent mutations including PIK3CA, NRAS, and HRAS remains uncertain since tumors without these mutations behaved equally aggressively with poor prognosis. Finally, two cases had concurrent FGFR4 mutations (V548M and V550L).

The MYODI gene encodes a nuclear protein that belongs to the basic helix-loop-helix (bHLH) family of transcription factors and the myogenic factors subfamily [22]. MYOD1 regulates muscle cell differentiation by inducing cell cycle arrest, a prerequisite for myogenic initiation. Mutation of Leu 122 to Arg in MYODl has been shown to confer reduced transcriptional activation at $M Y O D 1$ sites, together with enhanced binding to $M Y C$ sites [22].

In this study, more than half of the patients developed local recurrence and distant recurrences. Previous studies have suggested that pediatric MYODI-mutant rhabdomyosarcoma follows an aggressive course and poor prognosis [12-15]. In the study by Rekhi et al. [16], 7 of the 10 cases showed recurrence/metastasis and follow-up data showed 5 of the 10 cases were alive with disease. The current study confirms these prior smaller series, showing that $83 \%$ of pediatric patients died of the disease, with only $5 \%$ being alive with disease. Our findings further elucidate that MYOD1 mutant rhabdomyosarcoma has a poor prognosis in adults as well, with $55 \%$ of these patients succumbing of disease and $7 \%$ being alive with disease.

In conclusion, this study highlights the clinicopathologic features of MYOD1-mutant rhabdomyosarcoma, with morphologic spectrum ranging from spindle cell, sclerosing, and mixed histology (Fig. 5). Our findings also emphasize that MYODI-mutant rhabdomyosarcoma follows an aggressive clinical course, quite similar to alveolar rhabdomyosarcoma, with poor response to multimodality therapy and unfavorable outcome in both children and adults. Given that the MYODI mutant rhabdomyosarcoma is associated with aggressive biologic behavior, an argument can be made that MYODI mutation can be used for risk stratification as well as for subclassification of this distinct molecular group within the spindle/sclerosing rhabdomyosarcoma group (Table 2).

Acknowledgements The authors would like to thank Alyne Manzo for preparation of composite figures. They also thank the following pathologists and oncologists who kindly contributed case material and/ or clinical follow-up information when available: Dr. Ana Burga, Englewood, NJ; Dr. Angelica Putnam, Salt Lake City, UT; Dr. Kaisa Vepsäläinen, Finland; Dr. Turpin, Cincinnati, OH; Dr. Jianying Zeng, Brooklyn, NY; Dr. Gabriel Chamyan, Miami, FL; Dr. Douglas Fair, Salt Lake City, UT. This work was upported by PO1 CA047179-15A2 (CRA), P50 CA 140146-01 (CRA), Cycle for Survival (LW, CRA).

\section{Compliance with ethical standards}

Conflict of interest The authors declare that they have no conflict of interest.

\section{References}

1. Ognjanovic S, Linabery AM, Charbonneau B, Ross, JA. Trends in childhood rhabdomyosarcoma incidence and survival in the United States, 1975-2005. Cancer. 2009;115:4218-26.

2. Fletcher CD, Bridge JA, Hogendoorn PC, Mertens F. WHO classification of tumours of soft tissue and bone. Lyon: IARC Press; 2013. p. 251-306.

3. Oberlin O, Rey A, Lyden E, Bisogno G, Stevens, MC, Meyer WH, et al. Prognostic factors in metastatic rhabdomyosarcomas: results of a pooled analysis from United States and European cooperative groups. J Clin Oncol. 2008;26:2384-9. 
4. Cavazzana AO, Schmidt D, Ninfo V, Harms D, Tollot M, Carli $\mathrm{M}$, et al. Spindle cell rhabdomyosarcoma. A prognostically favorable variant of rhabdomyosarcoma. Am J Surg Pathol. 1992;16:229-35.

5. Leuschner I, Newton WA Jr, Schmidt D, Sachs N, Asmar L, Hamoudi A, et al. Spindle cell variants of embryonal rhabdomyosarcoma in the paratesticular region. A report of the Intergroup Rhabdomyosarcoma Study. Am J Surg Pathol. 1993; 17:221-30.

6. Folpe AL, McKenney JK, Bridge JA, Weiss SW. Sclerosing rhabdomyosarcoma in adults: report of four cases of a hyalinizing, matrix-rich variant of rhabdomyosarcoma that may be confused with osteosarcoma, chondrosarcoma, or angiosarcoma. Am J Surg Pathol. 2002;26:1175-83.

7. Mentzel T. Spindle cell rhabdomyosarcoma in adults: a new entity in the spectrum of malignant mesenchymal tumors of soft tissues. Der Pathol. 2010;31:91-6.

8. Mentzel T, Katenkamp D. Sclerosing, pseudovascular rhabdomyosarcoma in adults. Clinicopathological and immunohistochemical analysis of three cases. Virchows Arch. 2000;436:305-11.

9. Mentzel T, Kuhnen C. Spindle cell rhabdomyosarcoma in adults: clinicopathological and immunohistochemical analysis of seven new cases. Virchows Arch. 2006;449:554-60.

10. Szuhai K, de Jong D, Leung WY, Fletcher CD, Hogendoorn PC. Transactivating mutation of the MYOD1 gene is a frequent event in adult spindle cell rhabdomyosarcoma. J Pathol. 2014;232:300-7.

11. Kohsaka S, Shukla N, Ameur N, Lim D, Viale A, Socci ND, et al. A recurrent point mutation in MYOD1 defines a clinically aggressive subset of embryonal rhabdomyosarcoma associated with PI3K-AKT pathway mutations. Nat Genet. 2014;46:595-602.

12. Owosho AA, Huang SC, Chen S, Kashikar S, Estilo CL, Wolden SL, et al. A clinicopathologic study of head and neck rhabdomyosarcomas showing FOXO1 fusion-positive alveolar and MYOD1-mutant sclerosing are associated with unfavorable outcome. Oral Oncol. 2016;61:89-97.

13. Agaram NP, Chen CL, Zhang L, LaQuaglia MP, Wexler L, Antonescu CR. Recurrent MYOD1 mutations in pediatric and adult sclerosing and spindle cell rhabdomyosarcomas: evidence for a common pathogenesis. Genes Chromosomes Cancer. 2014;53:779-87.
14. Alaggio R, Zhang L, Sung YS, Huang SC, Chen CL, Bisogno $\mathrm{G}$, et al. A molecular study of pediatric spindle and sclerosing rhabdomyosarcoma: identification of novel and recurrent VGLL2related fusions in infantile cases. Am J Surg Pathol. 2016; 40:224-35

15. Owosho AA, Chen S, Kashikar S, Zhang L, Chen CL, Wexler LH, et al. Clinical and molecular heterogeneity of head and neck spindle cell and sclerosing rhabdomyosarcoma. Oral Oncol. 2016;58:e6-e11.

16. Rekhi B, Upadhyay P, Ramteke MP, Dutt A. MYOD1 (L122R) mutations are associated with spindle cell and sclerosing rhabdomyosarcomas with aggressive clinical outcomes. Mod Pathol. 2016;29:1532-40.

17. Chiles MC, Parham DM, Qualman SJ, Teot LA, Bridge JA, Ullrich F, et al. Sclerosing rhabdomyosarcomas in children and adolescents: a clinicopathologic review of 13 cases from the Intergroup Rhabdomyosarcoma Study Group and Children's Oncology Group. Pediatr Dev Pathol. 2004;7:583-94.

18. Antonescu CR, Sommer G, Sarran L, Tschernyavsky SJ, Riedel E, Woodruff JM, et al. Association of KIT exon 9 mutations with nongastric primary site and aggressive behavior: KIT mutation analysis and clinical correlates of 120 gastrointestinal stromal tumors. Clin Cancer Res. 2003;9:3329-37.

19. Cheng DT, Mitchell TN, Zehir A, Shah RH, Benayed R, Syed A, et al. Memorial Sloan Kettering-Integrated Mutation Profiling of Actionable Cancer Targets (MSK-IMPACT): a hybridization capture-based next-generation sequencing clinical assay for solid tumor molecular oncology. J Mol Diagn. 2015;17:251-64.

20. Mosquera JM, Sboner A, Zhang L, Kitabayashi N, Chen CL, Sung YS, et al. Recurrent NCOA2 gene rearrangements in congenital/infantile spindle cell rhabdomyosarcoma. Genes Chromosomes Cancer. 2013;52:538-50.

21. Shukla N, Ameur N, Yilmaz I, Nafa K, Lau CY, Marchetti A, et al. Oncogene mutation profiling of pediatric solid tumors reveals significant subsets of embryonal rhabdomyosarcoma and neuroblastoma with mutated genes in growth signaling pathways. Clin Cancer Res. 2012;18:748-57.

22. Van Antwerp ME, Chen DG, Chang C, Prochownik EV. A point mutation in the MyoD basic domain imparts c-Myc-like properties. Proc Natl Acad Sci USA. 1992;89:9010-4. 